\title{
ANALYSIS AND CONTROLLING OF HOPF BIFURCATION FOR CHAOTIC VAN DER POL-DUFFING SYSTEM
}

\author{
Ping Cai ${ }^{1,2}$, Jia-Shi Tang ${ }^{1}$, Zhen-Bo Li ${ }^{1}$ \\ ${ }^{1}$ College of Mechanical and Vehicle Engineering, Hunan University, Changsha 410082, \\ China \\ ${ }^{2}$ School of Mathematics and Statistics, Minnan Normal University, Zhangzhou 363000, \\ China \\ caiping0596@163.com
}

\begin{abstract}
Analysis and controlling of bifurcation for a class of chaotic Van der PolDuffing system with multiple unknown parameters are conducted. The stability of the equilibrium of the system is studied by using Routh-Hurwitz criterion, and the critical value of Hopf bifurcation is investigated. Based on the center manifold theory and normal form reduction, the stability index of bifurcation solution is given. Linear and nonlinear washout filter feedback controllers are designed respectively to control the bifurcation critical value and the amplitude of the limit cycle. Numerical simulation results are presented to illustrate analytical results found.
\end{abstract}

Key Words- chaotic Van der Pol-Duffing system, Hopf bifurcation, amplitude of limit cycle, controller

\section{INTRUDUCTION}

Bifurcation and chaos play an important role in the study of nonlinear science. In recent years, bifurcation control has attracted many researchers since chaos control is greatly developed. As is well known, Hopf bifurcation gives rise to limit circle, which has important theoretical significance and practical value. Recently, many research works on Hopf bifurcation control [1-4] and anti-control [5-7] have born great fruits. Hopf bifurcation control for high-dimensional system is more difficult. Various bifurcation control approaches have been proposed and used in many application, such as linear [4] and nonlinear feedback control [2]、washout filter feedback control [7], frequency domain analysis method [8] and normal form method [9].Consider the following chaotic Van der Pol-Duffing (ADVP) system

$$
\left\{\begin{array}{l}
\dot{x}_{1}=-v\left(x_{1}^{3}-\mu x_{1}-x_{2}\right) \\
\dot{x}_{2}=x_{1}-\alpha x_{2}-x_{3} \\
\dot{x}_{3}=\beta x_{2}
\end{array}\right.
$$


where, $\left(x_{1}, x_{2}, x_{3}\right) \in R^{3}$ are state variables, $\mu \in R, \alpha \geq 1, v>0, \beta>0$ are real parameters. When $\alpha=1$, system (1) reduces to a system equivalent to the classical Chua's differential equations with cubic nonlinearity. System (1) has rich dynamical behaviors including bifurcation and chaos. System (1) has attracted many researchers and has achieved considerable progress. Denis [10] studied the local codimension one, two, and three bifurcations of the system. Matouk [11] discussed the problem of chaotic synchronization. He [12] designed a kind of adaptive stabilizing controller, then the controlled system can convergent rapidly. As far as we known, control of Hopf bifurcation and amplitude of the limit cycle have not been performed to system (1). One of the representative approaches is applying washout filter-aided dynamic feedback controller, which preserve all the equilibrium points of the system. Washout filters have been widely applied in various bifurcating nonlinear systems $[7,13,14]$. In this paper, Hopf bifurcation and related control for the ADVP system through washout filters is focused on. Firstly, parameter critical value is derived through the analysis of stability of equilibrium point, and the stability index of bifurcation solution is also obtained based on the center manifold theory and normal form reduction. Secondly, linear and nonlinear of washout filter-aided dynamic feedback controllers are designed respectively to control Hopf bifurcation value and the amplitude of the limit cycle. Finally, numerical simulations are given to illustrate the effectiveness of the controller and the correctness of amplitude predictions.

\section{EXISTENCE OF HOPF BIFURCATION}

System (1) has three equilibrium: $E_{0}=(0,0,0)$ and $E_{ \pm}=( \pm \sqrt{\mu}, 0, \pm \sqrt{\mu})$ if $\mu>0$. Otherwise, system (1) has only one equilibrium $E_{0}=(0,0,0)$. Since $E_{+}$and $E_{-}$are symmetrically placed with respect to the y-axis, in what follows, Hopf bifurcation at $E_{-}$ can be obtained similarly at $E_{+}$. For simplicity, we only consider the Hopf bifurcation at $E_{0}$. Jacobian matrix of system (1) at $E_{0}$ has the form

$$
J\left(E_{0}\right)=\left(\begin{array}{ccc}
\mu \nu & v & 0 \\
1 & -\alpha & -1 \\
0 & \beta & 0
\end{array}\right)
$$

which corresponds to the characteristic equation

$$
\lambda^{3}+(\alpha-\mu v) \lambda^{2}+(\beta-v-\alpha \mu v) \lambda-\mu \beta v=0
$$


Tacking $\beta$ as the Hopf bifurcation parameter and supposing that Eq. (3) possesses a pair of pure imaginary eigenvalues $\lambda_{1,2}= \pm i \omega_{0}\left(\omega_{0}>0\right)$, we can reach

$$
\begin{aligned}
& \beta=\beta_{0}=\frac{v(\alpha-\mu \nu)(1+\alpha \mu)}{\alpha} \\
& \omega_{0}=\sqrt{\beta_{0}-v-\alpha \mu v}=\sqrt{\frac{-\mu v^{2}(1+\alpha \mu)}{\alpha}}
\end{aligned}
$$

Another negative root of Eq. (3) is $\lambda_{3}=\mu \nu-\alpha<0$. Therefore, a necessary condition for the system (1) to exhibit Hopf bifurcation at $E_{0}$ is

$$
-1<\alpha \mu<0
$$

Under these conditions, the transversality condition

$$
\operatorname{Re}\left(\left.\lambda^{\prime}(\beta)\right|_{\beta=\beta_{0}, \lambda=i \omega_{0}}\right)=\frac{-\alpha}{2 \omega_{0}^{2}+2(\alpha-\mu \nu)^{2}}<0
$$

is also satisfied. By using Routh-Hurwitz criterion, the equilibrium $E_{0}$ is stable for $\beta>\beta_{0}$, and loses its stability at critical point $\beta_{0}$. Therefore, system (1) undergoes Hopf bifurcation at the equilibrium $E_{0}$ based on Hopf bifurcation theory [15].

\section{ANALYSIS OF STABILITY OF HOPF BIFURCATION}

In this section, the stability of the bifurcating periodic solutions is analyzed using Hopf bifurcation theory.

By the linear transform $X=P Y$, where $Y=\left(y_{1}, y_{2}, y_{3}\right)^{T}$,

$$
P=\left(\begin{array}{ccc}
0 & 1 & 1 \\
\frac{\omega_{0}}{v} & -\mu & \frac{-\alpha}{v} \\
\frac{(-\alpha+\mu v) \omega_{0}}{v} & \frac{(\alpha-\mu v)(v+\alpha \mu v)}{\alpha v} & 1+\alpha \mu
\end{array}\right)
$$

then system (1) has the following normal form 


$$
\left\{\begin{array}{l}
\dot{y}_{1}=-\omega_{0} y_{2}+g_{1}\left(y_{1}, y_{2}, y_{3}\right) \\
\dot{y}_{2}=\omega_{0} y_{2}+g_{2}\left(y_{1}, y_{2}, y_{3}\right) \\
\dot{y}_{3}=(\mu v-\alpha) y_{3}+g_{3}\left(y_{1}, y_{2}, y_{3}\right)
\end{array}\right.
$$

where,

$$
\begin{aligned}
& g_{1}\left(y_{1}, y_{2}, y_{3}\right)=\frac{\alpha v^{2}(\alpha-2 \mu v)(1+\alpha \mu)\left(y_{2}+y_{3}\right)^{3}}{\omega_{0}\left(-\alpha^{3}+\mu v^{2}+2 \mu v \alpha^{2}\right)}, \\
& g_{2}\left(y_{1}, y_{2}, y_{3}\right)=-\frac{\alpha v\left(v-\alpha^{2}+2 \mu v \alpha\right)\left(y_{2}+y_{3}\right)^{3}}{-\alpha^{3}+\mu v^{2}+2 \mu v \alpha^{2}} \\
& g_{1}\left(y_{1}, y_{2}, y_{3}\right)=\frac{v^{2}(\alpha-\mu v)\left(y_{2}+y_{3}\right)^{3}}{-\alpha^{3}+\mu v^{2}+2 \mu v \alpha^{2}}
\end{aligned}
$$

According to the Hopf bifurcation theory, a curvature coefficient is expressed by

$$
\begin{aligned}
\sigma_{1}(0) & =\operatorname{Re}\left\{\frac{i}{2 \omega_{0}}\left(g_{20} g_{11}-2\left|g_{11}\right|^{2}-\frac{1}{3}\left|g_{02}\right|^{2}\right)+\frac{g_{21}}{2}\right\} \\
& =\frac{3 v \alpha\left(v-\alpha^{2}+2 \mu v \alpha\right)}{8\left(\alpha^{3}-\mu v^{2}-2 \mu v \alpha^{2}\right)}
\end{aligned}
$$

where

$$
\begin{aligned}
& g_{11}=0, \\
& g_{02}=0, \\
& g_{20}=0, \\
& g_{21}=\frac{3 v \alpha\left(v-\alpha^{2}+2 \mu v \alpha\right)}{4\left(\alpha^{3}-\mu v^{2}-2 \mu v \alpha^{2}\right)}+i \frac{3 v^{2} \alpha(\alpha-2 \mu v)(1+\alpha \mu)}{4 \omega_{0}\left(\alpha^{3}-\mu v^{2}-2 \mu v \alpha^{2}\right)}
\end{aligned}
$$

The above characteristic quantities can be calculated from Eq. (9). Denote $\eta=v-\alpha^{2}+2 \mu v \alpha$, If $\eta<0$, the periodic solutions, emanating from $E_{0}$ are stable and the bifurcation is supercritical. If $\eta>0$, the periodic solutions, emanating from $E_{0}$ are unstable and the bifurcation is subcritical. We take $v=3, \mu=0.25, \alpha=2[10]$, the system undergoes a Hopf bifurcation when the parameter $\beta$ crosses the critical value $\beta_{0}=2.0625$. With the curvature coefficient derived from (11) as $\sigma_{1}(0)=-0.55385<0$, 
the bifurcation is supercritical and the periodic solutions are stable. The bifurcation figure of uncontrolled system (1) is shown in Fig.1.

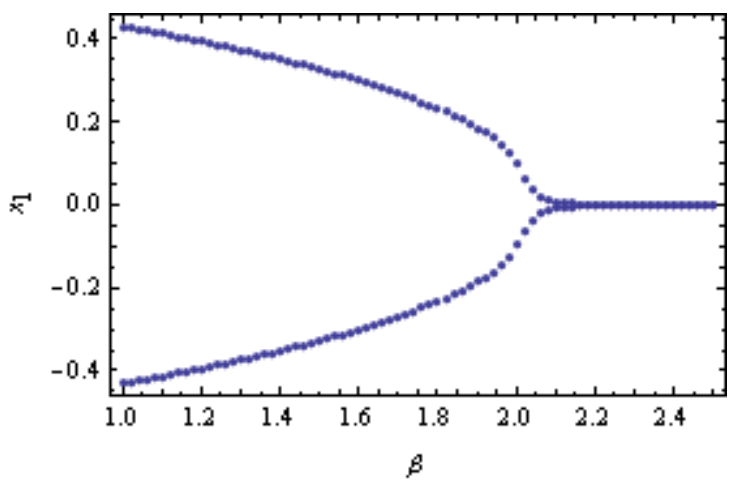

Figure 1. Bifurcation figure of uncontrolled system (1)

\section{CONTROL OF HOPF BIFURCATION}

In this section, the linear washout filter controller is designed to control Hopf bifurcation while not changing the stability of the bifurcating periodic solution. The controlled system is designed as follows

$$
\left\{\begin{array}{l}
\dot{x}_{1}=-v\left(x_{1}^{3}-\mu x_{1}-x_{2}\right) \\
\dot{x}_{2}=x_{1}-\alpha x_{2}-x_{3}+u \\
\dot{x}_{3}=\beta x_{2} \\
\dot{w}=x_{2}-d w
\end{array}\right.
$$

where, $w$ is controller variable and controller $u$ assumes the following form

$$
u=k\left(x_{2}-d w\right)
$$

where, $k$ is linear feedback gain. $d>0$, which guarantees the stability of the controller [16]. Obviously, the controller raises the dimension of system (1). Since system (1) is obtained from a circuit after a change of variables, parameters and a rescaling in time $[10,11]$, system (13) can be considered as the result of the transformation by adding another parallel resistor in the original circuit. The equilibrium of system (13) are $\widehat{E}_{0}=(0,0,0,0)$ and $\widehat{E}_{ \pm}( \pm \sqrt{\mu}, 0, \pm \sqrt{\mu}, 0),(\mu>0)$, so the original equilibrium were preserved. The associated characteristic equation of the linearized system (13) is

$$
\lambda^{4}+a_{1} \lambda^{3}+a_{2} \lambda^{2}+a_{3} \lambda+a_{4}=0
$$

where, 


$$
\begin{aligned}
& a_{1}=d+\alpha-\mu v-k, a_{2}=d \alpha-v+\beta-d \mu v-\alpha \mu v+k \mu v \\
& a_{3}=d \beta-d v-\alpha d \mu v-\beta \mu v, a_{4}=-\beta d \mu v
\end{aligned}
$$

The control gain $k$ and bifurcating critical value $\beta_{0}$ can be determined to the following relationship

$$
\begin{aligned}
& a_{i}>0, i=1,2,3,4, \\
& a_{1} a_{2}-a_{3}>0, \\
& a_{3}\left(a_{1} a_{2}-a_{3}\right)-a_{1}^{2} a_{4}=0
\end{aligned}
$$

We still let $v=3, \mu=0.25, \alpha=2$, and $d=0.1$. When we select $k=1$, the bifurcating critical value $\beta_{0}=3.82036$ is derived from (17), which is postpositional. If we select $k=-2, \beta_{0}=0.465142$ can be derived, which is prepositional. Thus the linear control term of washout filter-aided dynamic feedback controller can control Hopf bifurcation effectively, however not change stability of bifurcation. The bifurcation figures of the controlled system (13) when $k=1$ and $k=-2$ are shown in Fig.2 respectively.

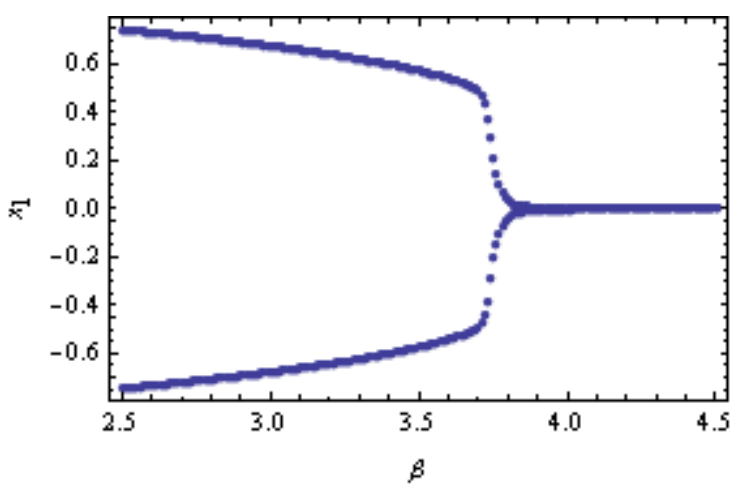

(a)

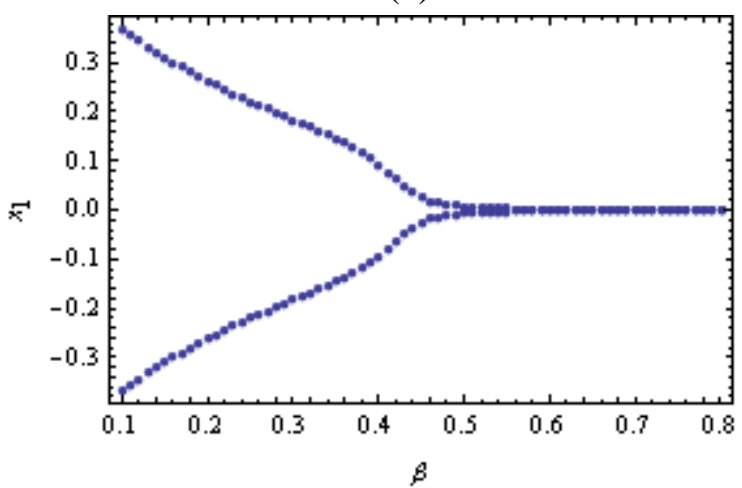

(b)

Figure 2. Bifurcation figure of the controlled system (13) 
(a) $k=1$; (b) $k=-2$

\section{AMPLITUDE CONTROL OF LIMIT CYCLES}

Amplitude control of limit cycle has received considerable attention [17,18]. In this section, the nonlinear washout filter controller is designed to control the amplitude of limit cycle emerging from the Hopf bifurcation in ADVP system. Under the control, the critical value $\beta_{0}$ is unchanged.

\subsection{Nonlinear control}

The controlled system is

$$
\left\{\begin{array}{l}
\dot{x}_{1}=-v\left(x_{1}^{3}-\mu x_{1}-x_{2}\right) \\
\dot{x}_{2}=x_{1}-\alpha x_{2}-x_{3}+u \\
\dot{x}_{3}=\beta x_{2} \\
\dot{w}=x_{2}-d w
\end{array}\right.
$$

where

$$
u=k_{n}\left(x_{2}-d w\right)^{3}
$$

This controller still does not affect the equilibrium structure of the original system (1). For simplicity, in the case $v=3, \mu=0.25, \alpha=2$, and $d=0.1$ are setted. At bifurcation value $\beta=\beta_{0}=2.0625$, the Jacobian matrix of system (18) has a pair of complex conjugate eigenvalues $\lambda_{1,2}= \pm 0.75 i$, and two real negative eigenvalues $\lambda_{3}=-2.75, \lambda_{4}=-0.1$. Then, using the transformation $X=P Y$, where

$$
\begin{aligned}
X=\left(x_{1}, x_{2}, x_{3}, w\right)^{T}, Y=\left(y_{1}, y_{2}, y_{3}, y_{4}\right)^{T}, & \\
P & =\left(\begin{array}{cccc}
0 & 1 & 1 & 0 \\
0.25 & 0.25 & -0.666667 & 0 \\
-0.6875 & 0.6875 & 0.5 & 0 \\
-0.283843 & 0.371179 & 0.251572 & 1
\end{array}\right)
\end{aligned}
$$

the controlled system (18) now reads as a canonical form 


$$
\left\{\begin{array}{l}
\dot{y}_{1}=-0.75 y_{2}+g_{1}\left(y_{1}, y_{2}, y_{3}, y_{4}, k_{n}\right) \\
\dot{y}_{2}=0.75 y_{2}+g_{2}\left(y_{1}, y_{2}, y_{3}, y_{4}, k_{n}\right) \\
\dot{y}_{3}=-2.75 y_{3}+g_{3}\left(y_{1}, y_{2}, y_{3}, y_{4}, k_{n}\right) \\
\dot{y}_{4}=-0.1 y_{4}+g_{4}\left(y_{1}, y_{2}, y_{3}, y_{4}, k_{n}\right)
\end{array}\right.
$$

where,

$$
\begin{aligned}
& g_{1}\left(y_{1}, y_{2}, y_{3}, y_{4}, k_{n}\right)=-2.5846\left(y_{2}+y_{3}\right)^{3} \\
& +0.2769 k_{n}\left(0.2784 y_{1}+0.2129 y_{2}-0.6918 y_{3}-0.1 y_{4}\right)^{3}, \\
& g_{2}\left(y_{1}, y_{2}, y_{3}, y_{4}, k_{n}\right)=-1.4769\left(y_{2}+y_{3}\right)^{3} \\
& +1.0154 k_{n}\left(0.2784 y_{1}+0.2129 y_{2}-0.6918 y_{3}-0.1 y_{4}\right)^{3}, \\
& g_{3}\left(y_{1}, y_{2}, y_{3}, y_{4}, k_{n}\right)=-1.5231\left(y_{2}+y_{3}\right)^{3} \\
& -1.0154 k_{n}\left(0.2784 y_{1}+0.2129 y_{2}-0.6918 y_{3}-0.1 y_{4}\right)^{3}, \\
& g_{4}\left(y_{1}, y_{2}, y_{3}, y_{4}, k_{n}\right)=0.1977\left(y_{2}+y_{3}\right)^{3} \\
& -0.0428 k_{n}\left(0.2784 y_{1}+0.2129 y_{2}-0.6918 y_{3}-0.1 y_{4}\right)^{3}
\end{aligned}
$$

\subsection{Relationship between amplitude of limit cycles and control gain}

The parameters are fixed as above $v=3, \mu=0.25, \alpha=2$, and we can calculate $\alpha^{\prime}(0)=\operatorname{Re}\left(\lambda^{\prime}\left(\beta_{0}\right)\right)=-0.12308, \sigma_{1}(0)=-0.55385+0.01351 k_{n} \quad . \quad$ Obviously, $\sigma_{1}(0)$ degrade into the curvature coefficient of the uncontrolled system when $k_{n}=0$. For ensuring the stability of the bifurcated limit cycles, it should be $k_{n}<41$. Therefore, the amplitude approximation for $\beta<\beta_{0}=2.0625$ and $|2.0625-\beta|<<1$ is

$$
r=\sqrt{-\frac{\alpha^{\prime}(0)}{\sigma_{1}}\left(\beta-\beta_{0}\right)}=0.4714 \sqrt{\frac{1}{1-0.0244 k_{n}}(2.0625-\beta)}
$$

The $k_{n}-r$ curve is shown in Fig. 3 with $\beta=1.9625$. Fig. 3 shows the amplitude of the limit cycle $r$ decreases as the control gain $k_{n}$ decreases. And the controller can make the amplitude sufficiently small. Fig. 3 also shows the relative error of the approximated solution of amplitude with numerical solution is no more than $3.8 \%$ when $k_{n}<20$, 
which has high accuracy. It should be pointed out that Eq. (23) describes the amplitude of the state variable $x_{1}$ of the controlled system. One can change the linear transformation (20) to describe the amplitude of the other state variables.

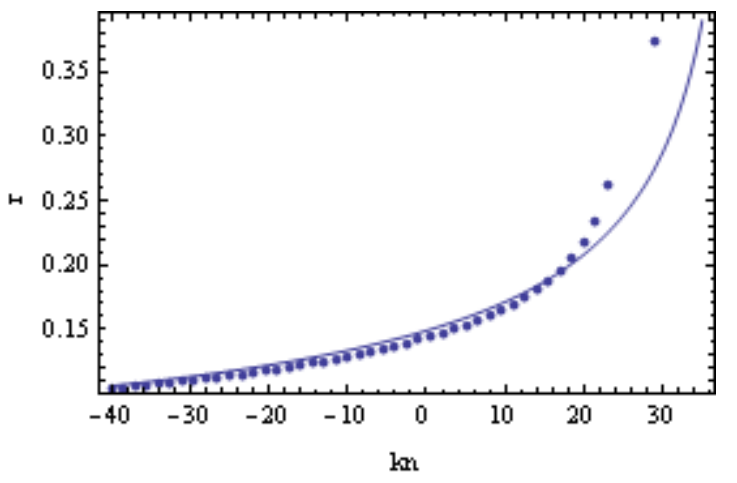

Figure 3. $k_{n}-r$ curve for the controlled system (18)

. . . . . numerical solution, ___ approximated solution

\section{CONCLUSIONS}

The nonlinear dynamical behaviors of the equilibrium of the ADVP system are discussed. The existence and stability of the limit cycle are analyzed in virtue of Hopf bifurcation theory. The linear control term of washout filter-aided dynamic feedback controller has been used to control Hopf bifurcation but not changing the stability. The nonlinear control term of washout filter-aided controller has been used to control the amplitude of the limit cycle but not changing the bifurcating critical value. The amplitude approximations in terms of control gains are derived from the center manifold theory and normal form reduction, which can also effectively predict the amplitude of limit cycles. Numerical simulation results are presented to illustrate the correctness and efficiency of the analytical results.

Acknowledgement- The project was supported by the National Natural Science Foundation of China (11172093, 11372102)

\section{REFERENCES}

1. S. Liu, H. R. Liu, Y. Wen, etal, Hopf bifurcation control in a coupled nonlinear relative rotation dynamical system, Acta Physica Sinica 59, 5223-5228, 2010 (in Chinese). 
2. P. Yu, G. R. Chen, Hopf bifurcation control using nonlinear feedback with polynomial functions, International Journal of Bifurcation and Chaos 14, 1683-1704, 2004.

3. Z. R. Liu, K. W. Chung, Hybrid control of bifurcation in continuous nonlinear dynamical systems coupled chaotic systems, International Journal of Bifurcation and Chaos 15, 3895-3903, 2005.

4. S. H. Liu, J. S. Tang, Linear feedback control of Hopf bifurcation in Langford system, Acta Physica Sinica 56, 3145-3151, 2007 (in Chinese).

5. S. H. Liu, J. S.Tang, Anti-control of Hopf bifurcation at zero equilibrium of 4D Qi system, Acta Physica Sinica 57, 6162-6168, 2008 (in Chinese).

6. Z. S. Lu, L. X. Duan, Anti-Control of Hopf Bifurcation in the Chaotic Liu System with Symbolic Computation, Chinese Physics Letters 26, 050504, 2009.

7. Z. S. Cheng, J. D. Cao, Anti-control of Hopf bifurcation for Chen's system through washout filters, Neurocomputing 73, 3139-3146, 2010.

8. Z. H. Wang, H. Y. Hu, Delay-Independent Stability Of Retarded Dynamic Systems Of Multiple Degrees Of Freedom, Journal of Sound and Vibration 226, 57-81, 1999.

9. K. Wei, A. J. Krener, Extended Quadratic Controller Normal Form and Dynamic State Feedback Linearization of Nonlinear Systems, SIAM Journal on Control and Optimization 30, 1319-1337, 1992.

10. D. C. Braga, L. F. Mello, M. Messias, Bifurcation Analysis of a Van der PolDuffing Circuit with Parallel Resistor, Mathematical Problems in Engineering 149563, 2009.

11. A. E. Matouk, H. N. Agiza, Bifurcations, chaos and synchronization in ADVP circuit with parallel resistor, Journal of Mathematical Analysis and Applications 341, 259-269, 2008.

12. H. L. He, J. J. Tu, P. Xiong, Parameter adaptive stabilization for chaotic ADVP systems based on back-stepping approach, Journal of Huazhong University of Science and Technology (Natural Science Edition) 39, 92-94, 2011 (in Chinese).

13. C. X. Liang, J. S. Tang, S. H. Liu, Hopf bifurcation control of a hyperchaotic Circuit system, Communications in Theoretical Physics 52, 457-462, 2009.

14. Q. Dinga, D. L. Wang, Flutter Control of a Two-dimensional Airfoil Using Washout Filter Technique, CHINESE JOURNAL OF AERONAUTICS 18, 130-137, 2005.

15. B. D. Hassard, N. D. Kazarinoff, Y. Wan, Theory and Applications of Hopf Bifurcation, Cambridge Univ., London, 1981.

16. H. O. Wang, E. H. Abed, Bifurcation control of a chaotic system, Automatica 3, 213-1226, 1995.

17. C. Yan, S. H. Liu, J.S. Tang, Y. M. Meng, Amplitude control of limit cycles in Langford system, Chaos, Solitons and Fractals 42, 335-340, 2009.

18. K. J. Ouyang, J. S. Tang, C. X. Liang, Amplitude control of limit cycle in van der Pol-Duffing system, Chinese Physics B 18, 4748-4753, 2009. 